\title{
Towards Ultra-Tough Oxide Glasses with Heterogeneities by Consolidation of Nanoparticles
}

\author{
Yanming Zhang, Liping Huang, Yunfeng Shi* \\ Department of Materials Science and Engineering, Rensselaer Polytechnic Institute, $1108^{\text {th }}$ \\ Street, Troy, New York 12180, USA \\ *shiy2@rpi.edu
}

We prepared heterogeneous alumina-silicate glasses by consolidating nanoparticles using molecular dynamics simulations. Consolidated glasses from either low alumina content alumina-silicate glasses or high alumina content alumina-silicate glasses show significantly improved ductility around consolidation pressure of $\sim 3$ GPa. The introduced structural heterogeneities, namely over-coordinated network formers and their neighboring oxygen atoms, are identified as plasticity carriers due to their high rearrangement propensity. In addition, consolidated oxide glass from both $23.4 \mathrm{Al}_{2} \mathrm{O}_{3} 76.6 \mathrm{SiO}_{2}$ and $73.1 \mathrm{Al}_{2} \mathrm{O}_{3} 26.9 \mathrm{SiO}_{2}$ nanoparticles show improved flow strength (up to 1GPa) due to the introduction of chemical heterogeneities. Last but not least, apparent hardening behavior appears upon cold work in consolidated glasses, with an increase of yield strength from $\sim 3.3 \mathrm{GPa}$ to $\sim 6.4 \mathrm{GPa}$. This method is a big advancement toward ultra-strong and ultra-tough glasses by breaking the structure, composition and size limitations in traditional melt-quench process.

Keywords: Oxide glasses; strength; ductility; work hardening ability; nanoscale heterogeneities; molecular dynamics simulations 


\section{INTRODUCTION}

Oxide glasses are widely used in energy, electronic and engineering applications thanks to their intriguing optical, thermal and chemical properties. ${ }^{1-3}$ However, glasses are generally brittle due to their low shear-flow propensity and poor crackpropagation resistance. ${ }^{4,5}$ Such brittleness is the stumbling block of current commercial glass products since it will decrease the damage tolerance and practical strength ${ }^{6}$. Hence, there is a strong drive to develop ultra-tough oxide glasses.

Many efforts have been made to toughen amorphous oxides in the past. The most widely used strategy is surface strengthening, which can inhibit crack propagation with the pre-built compressive stress near surface layer. However, such surface treatments are not universally applicable. For example, thermal tempering ${ }^{7}$ can't be applied to low-thermal-expansion oxide glasses; chemical tempering ${ }^{8,9}$ is restricted to glasses with mobile ions. More importantly, surface strengthening is exclusively effective in thin-slab sample, as the internal cracks will cause catastrophic failure in bulk.

Apart from developing compressive stress, tough oxide glasses can also be obtained by increasing ductility. On one hand, new glass systems like cesium aluminoborate, binary aluminosilicate and pure alumina glasses ${ }^{10-13}$ have shown potential to possess intrinsically high ductility. For instance, amorphous alumina was found to exhibit viscous creep at room temperature, which leads to exceptional ductility up to $100 \%$. However, the glass forming ability of ductile amorphous oxides is usually very low, hence we cannot easily synthesize them by traditional melt-quenching processes. Even with special levitation synthetic techniques, the size of the obtained amorphous alumina is still restricted to submicron. On the other hand, ductility of existing amorphous oxides can be enhanced using hydrostatical pressure treatments, either at room temperature or during meltquenching process ${ }^{14-16}$. The key of this technique is to introduce structural heterogeneities as plasticity carriers, such as five-fold silicon atoms in silica glasse ${ }^{17,18}$. But the plastic carriers are very challenging to create, since it usually requires extremely high pressure (>10 GPa for silica glass) to display substantial 
ductility. In the meanwhile, considerable strength reduction is commonly observed after applying hydrostatical pressure, hence limits the toughening effects. Therefore, besides introducing structural heterogeneities, the bonding topology should also be optimized to maintain the high strength.

Based on the discussion above, to satisfy the escalating need in stronger and more damage-resistant oxide glasses, we should develop more powerful, feasible and universal toughening strategies. To this end, Zhang et al ${ }^{19}$ prepared tough silica glass with significantly reduced pressure by consolidating glassy nanoparticles. This consolidation technique may be a universal route to improve both the intrinsic ductility and strength simultaneously.

Herein, we systematically investigated the toughening effects of consolidation technique with nanoparticles on various typical oxide glasses using molecular dynamics (MD) simulations. We found that the brittle to ductile transition in intrinsically brittle glass (with low alumina content) requires $\sim 3 \mathrm{GPa}$ by consolidating nanoparticles, much lower than that for compressing bulk sample (over $7 \mathrm{GPa}$ ). For intrinsically ductile yet low-formable glasses (high-alumina content and pure alumina), $\sim 3 \mathrm{GPa}$ is already sufficient to obtain considerable ductility in bulk samples by consolidating nanoparticles. Interestingly, up to $1 \mathrm{GPa}$ enhancement of flow strength is obtained in consolidated heterogenous glasses owing to the unique and stable bonding among atoms in glassy nanoparticles. Last but not least, apparent work hardening ability is achieved in the consolidate glasses, with yield strength increasing from $\sim 3.3 \mathrm{GPa}$ to $\sim 6.4 \mathrm{GPa}$ after $40 \%$ cold work. We believe that the method delineated here can break the structure, composition and size limitation in traditional glass preparation route, and will be regarded as a universal and feasible technique toward ultra-tough oxide glasses.

\section{SIMULATION SETUP}


Classical molecular dynamic simulations were carried out in LAMMPS package $^{24}$ (https://lammps.sandia.gov/). The equations of motion were integrated by Velocity-Verlet algorithm with a time step of $0.8 \mathrm{fs}$. To control the pressure and temperature of the system, Nose-Hoover barostat and thermostat ${ }^{25,26}$ were used. To mimic the behavior of binary aluminosilicate glasses, a modified version of the Buckingham potential ${ }^{27}$ is used, which can accurately reproduce the structure and mechanical properties over a wide composition range (as in Figure S4). The shortrange cutoff is set as $0.8 \mathrm{~nm}$. The long-range Columbic interaction which cut at 1 $\mathrm{nm}$ was calculated via the Wolf truncation method $^{28}$ to increase the computational efficiency.

Bulk samples with varying compositions were quenched from wellequilibrated high temperature $(3600 \mathrm{~K})$ melt to room temperature $(300 \mathrm{~K})$ with a rate about $4 \mathrm{~K} / \mathrm{ps}$ under zero pressure. Nanoparticles with uniform size are then carved from this bulk glass. Charge balance of the entire samples are maintained by deleting surface atoms of nanoparticles. The size of nanoparticles might be slightly adjusted to maintain the same nominal concentration. Proceeding consolidation, relaxation of nanoparticles is conducted at room temperature under constant volume for $\sim 1 \mathrm{~ns}$ to avoid any artificial periodicity. The entire simulation box is then subjected to consolidation following a temperature and pressure profile in Figure S5. The $1^{\text {st }}$ neighbor cutoff for Si-O and Al-O is $0.22,0.24 \mathrm{~nm}$, respectively as in the RDF curves in Figure S6. For starting nanoparticle size ranging from 1 to $8 \mathrm{~nm}$, the resulting glassy sample after consolidation is around $10 \mathrm{~nm}$ by $10 \mathrm{~nm}$ by $20 \mathrm{~nm}$ (atoms population 200000). The sample size is around $20 \mathrm{~nm}$ by $20 \mathrm{~nm}$ by $20 \mathrm{~nm}$ when nanoparticle size is above $8 \mathrm{~nm}$ (atoms population $\sim 1$ million). The sample is sufficiently long and not slender enough to trigger the known size/shape effect. ${ }^{29,30}$ Uniaxial tension testes with an engineering strain rate of $1.25 \mathrm{~ns}^{-1}$ were carried out under room temperature to investigate the mechanical properties. For ductile oxide glasses, the flow stress is averaged from $30 \%$ to $65 \%$. Periodic boundary conditions were applied in all three directions. The visualization software OVITO ${ }^{31}$ was used to generate simulation snapshots, animations and local 
atomic strain/stress was calculated based on method proposed by Falk and Langer $^{32}$.

\section{RESULTS AND DISCUSSIONS}

To prepare bulk samples, the nanoparticles are consolidated at different temperature and pressure followed the profile in Method section. Figure 1 illustrates the sample morphology consolidated under different pressure after returning back to zero pressure and room temperature $(300 \mathrm{~K})$. To introduce nanoscale chemical heterogeneities inside the oxide glasses, a mixture of nanoparticles with two different concentrations are used for consolidation. In this regard, the volume fraction of starting nanoparticles is carefully adjusted to maintain the same nominal composition. The degree and distribution of chemical heterogeneities can be tuned in a systematic way by changing the size and composition of the starting nanoparticles. In contrast, if only one type of nanoparticles were used for consolidation, the obtained glasses are thought to be free of chemical heterogeneities. For convenience, the consolidated glasses with chemical heterogeneities, the consolidated glasses without chemical heterogeneities and the compressed as-quenched glasses are termed binary consolidated glasses (BCS), mono consolidated glasses (MCS), compressed glasses (CP), respectively. The mechanical properties of obtained oxide glassed are investigated by conducting uniaxial tension tests with an engineering strain rate of $1.25 \mathrm{~ns}^{-1}$.

First, we systematically investigated the mechanical properties of consolidated glasses with a nominal composition of $23.4 \mathrm{Al}_{2} \mathrm{O}_{3} 76.6 \mathrm{SiO}_{2}$. The as-quenched glass is free of pores inside, yet still exhibits generic brittle behavior as in Figure 2 (b). To prepare glasses with chemical heterogeneities, both 8-nm amorphous alumina and silica are consolidated at room temperature using various pressure. With low applied pressure ( $\sim 5 \mathrm{GPa})$, large pores are observable in the consolidated glasses. The densities of consolidated glasses are provided in Figure S1. As the applied 
consolidation pressure increases, the BCS exhibit apparent brittle to ductile (BTD) transition with a critical pressure of $\sim 10 \mathrm{GPa}$. Interestingly, by introducing chemical heterogeneities, the flow strength of consolidated glass is enhanced from 6.4 GPa to 7.2 GPa. Such substantial improvement in strength will not sacrifice ductility, which is of vital importance for toughness optimization. We then reduced the size of both silica and alumina starting glassy nanoparticles from $8 \mathrm{~nm}$ to $1 \mathrm{~nm}$, Figure 2 (c). Despite the existence of pores insides, improved ductility can be achieved in BCS samples when applying $\sim 3$ GPa pressure, much lower than over 7 GPa needed for compressing bulk samples albeit the bulk sample is indeed defectsfree (Figure S2). The critical pressure observed here is slightly higher than that of pure silica $(2.4 \mathrm{GPa})$, which might originate from the inhibition of pore healing by the high elastic moduli of amorphous alumina. Note that the flow strength of 1-nmBCS is lower than that of the 8-nm-BCS, but still higher than CP sample. The mechanism for enhanced strength in the heterogeneous consolidated glasses will be discussed later. When subjecting to higher consolidation temperature, CS samples display both improved ductility and strength as shown in Figure 2(d). Specifically, the BCS consolidated under $1500 \mathrm{~K}$ and $3.2 \mathrm{GPa}$ possesses comparable strength as the as-quenched glass, but over 2 times enhancement in ductility. Finally, different compositions of starting nanoparticles are consolidated at 3.2 GPa as in Figure 2(e), where subtle difference in critical pressure is observable as the starting nanoparticle composition changes. Besides, BCS prepared from a mixture of pure glassy alumina and silica are found to be slightly stronger than those made from 23. $4 \mathrm{Al}_{2} \mathrm{O}_{3} 76.6 \mathrm{SiO}_{2}$ nanoparticles.

Using this consolidation technique, we then prepared two types of low-formable ductile oxide glasses with different starting materials, where final morphologies can be found in Figure 3(a). For high alumina content binary aluminosilicate glass with nominal composition of $73.1 \mathrm{Al}_{2} \mathrm{O}_{3} 26.9 \mathrm{SiO}_{2}$, the as-quenched sample exhibits generic ductile behavior, Figure 3(b). When preparing glass with the same nominal composition by consolidating a mixture of 2-nm glassy alumina and silica upon different pressure at room temperature, we found $\sim 3 \mathrm{GPa}$ to be the critical pressure 
to obtain sufficient ductility for BCS. Meanwhile, the BCS exhibits slightly enhanced flow strength as compared to both $\mathrm{CP}$ and as-quenched glass. We also made the sample using 2-nm glassy $73.1 \mathrm{Al}_{2} \mathrm{O}_{3} 26.9 \mathrm{SiO}_{2}$ nanoparticles at room temperature under various pressure. Similarly, as applied pressure increases, enhancement in ductility is observed, where the critical pressure is again $\sim 3 \mathrm{GPa}$. For pure alumina, the as-quenched sample also exhibits superior intrinsic ductility as in Figure 3(c). To prepare amorphous alumina, either $2 \mathrm{~nm}$ glassy nanoparticles or crystalline nanoparticles are consolidated at room temperature. Due to the high intrinsic ductility of amorphous alumina, the improvement in ductility as applied pressure increases for consolidated alumina is mainly due to the closing of pores, as proved by the enhancement in density (Figure S3). Note that the critical pressure to obtain sufficient ductility $(\sim 40 \%)$ in those two consolidated glasses is less than 3 GPa. However, the glasses prepared with glassy nanoparticles show obviously improved ductility at the same applied pressure.

Apart from the obtained ductile behavior for both low and high aluminosilicate glasses, we also investigated the effects of chemical heterogeneities on the strength of consolidated glass. When consolidating with glassy alumina and silica, the flow strength of $23.4 \mathrm{Al}_{2} \mathrm{O}_{3} 76.6 \mathrm{SiO}_{2}$ first increases with the starting nanoparticle size, then reaches plateau as the size exceeds $8 \mathrm{~nm}$, see Figure 4(a). This plateau value $(\sim 7.2 \mathrm{GPa})$ is very close to the theoretical flow strength of glass composited with densified amorphous silica and alumina $(\sim 7.3 \mathrm{GPa})$. Besides pure amorphous alumina, we also prepared the sample using vitreous silica and various binary aluminosilicate nanoparticles as starting materials; we maintained the same nominal composition by carefully adjusting the volume fraction of silica nanoparticles. The flow strength is found to increase with volume fraction of silica nanoparticles, since silica glass display higher flow strength ( $\sim 8 \mathrm{GPa})$, Figure 4(b). Finally, we prepared glasses over a wide range of nominal composition using 8nm-glassy alumina and silica under $300 \mathrm{~K}$ and $19.2 \mathrm{GPa}$. Compared with compressing the as-quenched glasses, enhancement in flow strength is obtained for glasses consolidated from nanoparticles over a large nominal composition range, 
reaching $1 \mathrm{GPa}$ in $50 \mathrm{Al}_{2} \mathrm{O}_{3} 50 \mathrm{SiO}_{2}$, Figure $4(\mathrm{c})$. We attribute the enhanced strength to the preserved unique stable bonding in starting nanoparticles through introducing chemical heterogeneities. This stable bonding is confirmed by the low potential energy of consolidated glasses as in Figure 4(d). Note that such bonding cannot be obtained in traditional melt-quenching process since it is considerably deteriorated due to entropic effect, especially for $50 \mathrm{Al}_{2} \mathrm{O}_{3} 50 \mathrm{SiO}_{2}$. Consequently, by introducing both structural and chemical heterogeneities across the samples, we realized simultaneous enhancement of strength and ductility in consolidated glasses.

Furthermore, we observed more apparent hardening ability in the consolidated glasses by conducting loading-reloading-unloading mechanical tests, Figure 5. When compressing as-quenched glass $\left(23.4 \mathrm{Al}_{2} \mathrm{O}_{3} 76.6 \mathrm{SiO}_{2}\right)$, the yield strength of the original samples is $\sim 3 \mathrm{GPa}$, which increases to $\sim 4.2,5.4,5.8 \mathrm{GPa}$ after cold work corresponding with $8 \%, 20 \%, 40 \%$ strain. In contrast, consolidated glass prepared with 8 -nm amorphous alumina and silica nanoparticles originally yields at $\sim 3.3 \mathrm{GPa}$, which is significantly enhanced to $4.9,6.2,6.4 \mathrm{GPa}$ when cold working at $8 \%, 20 \%$ and $40 \%$ strain, respectively. Such more obvious hardening ability is due to their superior low strength introduced by chemical heterogeneities.

It is well known that over-coordinated atoms appear after pressure treatment ${ }^{20,21}$ and are found to be responsible for enhancement in ductility for silica glass ${ }^{18,19,22}$. Therefore, to understand the structural origin of superior ductility in the binary aluminosilicate glasses, we first analyzed the correlation between plasticity and over-coordinated atoms. We found that the plasticity of binary oxide glasses is increased with the population of oxygen atoms bonded with over-coordinated cations, regardless of composition and processing routes, as shown in Figure 6(a). Theoretically, plastic deformation is realized by the incremental bond switching events; hence, we further examine the rearrangement propensity of oxygen atoms with different coordination environment in the remaining plastic deformation after unloading. As shown in Figure 6 (b), the oxygen atoms bonded with those overcoordinated cations $(\mathrm{CN}>=5)$ display relatively high rearrangement propensity of 
$\sim 40 \%$, which renders them acting as plasticity carriers during plastic deformation. By consolidating nanoparticles, a larger population of plasticity carriers is generated, hence the BTD transition is triggered with low pressure, Figure 6(c). The work hardening behavior can also be understood by the relaxation of plasticity carriers as in Figure 7. Before loading, the population is $\sim 25 \%$ for compressed bulk sample. The plasticity carrier population is reduced to $21 \%, 13 \%, 11 \%$ as cold worked at $8 \%, 20 \%$ and $40 \%$, respectively. Similarly, the population of plasticity carriers is $28 \%$ for consolidated glasses proceeding cold work. By cold work with $8 \%, 20 \%, 40 \%$ strain, the population of plasticity carriers is reduced to $25 \%, 22 \%$, $20 \%$, respectively. Therefore, the stress facilitated relaxation of plasticity carriers is the origin for the observed hardening behavior, similar behavior is observed in consolidated silica glass ${ }^{19}$ and rejuvenated metallic glasses ${ }^{23}$.

In conclusion, the consolidated glasses with nanoparticles enables synergistic optimization of structural and chemical heterogeneities, which leads to simultaneous enhancement in strength and ductility. For binary aluminosilicate and pure alumina glass, ductile glasses in bulk size can be obtained by consolidating nanoparticles with relatively low pressure ( $\sim 3 \mathrm{GPa}$ ). Over-coordinated cations and their neighboring oxygen atoms are identified as the plasticity carriers in the binary glasses due to their high rearrangement propensity. The introduction of chemical heterogeneities results in higher flow strength due to preserved stable bonding. Ductile binary glasses exhibit apparent hardening ability, especially for oxide glasses with chemical heterogeneities. The proposed consolidation technique will shed light on designing stronger and more damage-resistant oxide glasses, paving the way for future applications. 


\section{REFERENCE}

1. Huang, L., Durandurdu, M. \& Kieffer, J. Transformation pathways of silica under high pressure. Nat. Mater. 5, 977-981 (2006).

2. Shi, Y. \& Falk, M. L. Strain Localization and Percolation of Stable Structure in Amorphous Solids. Phys. Rev. Lett. 95, (2005).

3. Yin, Z., Hannard, F. \& Barthelat, F. Impact-resistant nacre-like transparent materials. Science 364, 1260-1263 (2019).

4. Shi, Y., Luo, J., Yuan, F. \& Huang, L. Intrinsic ductility of glassy solids. J. Appl. Phys. 115, 043528 (2014).

5. Wondraczek, L. et al. Towards Ultrastrong Glasses. Adv. Mater. 23, 4578-4586 (2011).

6. Deng, B., Luo, J., Harris, J. T., Smith, C. M. \& McKenzie, M. E. Molecular dynamics simulations on fracture toughness of A12O3-SiO2 glass-ceramics. Scr. Mater. 162, 277-280 (2019).

7. Uhlmann, D. Elasticity and Strength in Glasses: Glass: Science and Technology. (Elsevier, 2012).

8. Gy, R. Ion exchange for glass strengthening. Mater. Sci. Eng. B 149, 159-165 (2008).

9. Wang, M. et al. Ion exchange strengthening and thermal expansion of glasses: Common origin and critical role of network connectivity. J. Non-Cryst. Solids 455, 70-74 (2017).

10. Luo, J., Vargheese, K. D., Tandia, A., Harris, J. T. \& Mauro, J. C. Structural origin of intrinsic ductility in binary aluminosilicate glasses. J. Non-Cryst. Solids 452, 297-306 (2016).

11. Rosales-Sosa, G. A., Masuno, A., Higo, Y. \& Inoue, H. Crack-resistant Al2O3-SiO2 glasses. Sci. Rep. 6, 23620 (2016).

12. Frankberg, E. J. et al. Highly ductile amorphous oxide at room temperature and high strain rate. Science 366, 864-869 (2019).

13. Januchta, K. et al. Breaking the Limit of Micro-Ductility in Oxide Glasses. Adv. Sci. 6, 1901281 (2019).

14. Huang, L., Yuan, F., Guerette, M., Zhao, Q. \& Sundararaman, S. Tailoring structure and properties of silica glass aided by computer simulation. J. Mater. Res. 32, 174-182 (2017).

15. Januchta, K. et al. Discovery of Ultra-Crack-Resistant Oxide Glasses with Adaptive Networks. Chem. Mater. 29, 5865-5876 (2017).

16. Shi, Y., Katz, M. B., Li, H. \& Falk, M. L. Evaluation of the Disorder Temperature and FreeVolume Formalisms via Simulations of Shear Banding in Amorphous Solids. Phys. Rev. Lett. 98, 185505 (2007).

17. Guerette, M. et al. Structure and Properties of Silica Glass Densified in Cold Compression and Hot Compression. Sci. Rep. 5, 15343 (2015).

18. Yuan, F. \& Huang, L. Brittle to Ductile Transition in Densified Silica Glass. Sci. Rep. 4, 5035 (2014). 
19. Zhang, Y., Huang, L. \& Shi, Y. Silica Glass Toughened by Consolidation of Glassy Nanoparticles. Nano Lett. (2019) doi:10.1021/acs.nanolett.9b01634.

20. Wakabayashi, D., Funamori, N., Sato, T. \& Taniguchi, T. Compression behavior of densified SiO2 glass. Phys. Rev. B 84, (2011).

21. Liang, Y., Miranda, C. R. \& Scandolo, S. Mechanical strength and coordination defects in compressed silica glass: Molecular dynamics simulations. Phys. Rev. B 75, (2007).

22. Luo, J. et al. Size-Dependent Brittle-to-Ductile Transition in Silica Glass Nanofibers. Nano Lett. 16, 105-113 (2016).

23. Pan, J., Ivanov, Y. P., Zhou, W. H., Li, Y. \& Greer, A. L. Strain-hardening and suppression of shear-banding in rejuvenated bulk metallic glass. Nature 578, 559-562 (2020).

24. Plimpton, S. Fast Parallel Algorithms for Short-Range Molecular Dynamics. J. Comput. Phys. 117, 1-19 (1995).

25. Nosé, S. A unified formulation of the constant temperature molecular dynamics methods. $J$. Chem. Phys. 81, 511-519 (1984).

26. Nosé, S. A molecular dynamics method for simulations in the canonical ensemble. Mol. Phys. 52, 255-268 (1984).

27. Sundararaman, S., Huang, L., Ispas, S. \& Kob, W. New optimization scheme to obtain interaction potentials for oxide glasses. J. Chem. Phys. 148, 194504 (2018).

28. Wolf, D., Keblinski, P., Phillpot, S. R. \& Eggebrecht, J. Exact method for the simulation of Coulombic systems by spherically truncated, pairwise $\mathrm{r}-1$ summation. J. Chem. Phys. 110, 8254-8282 (1999).

29. Sopu, D., Foroughi, A., Stoica, M. \& Eckert, J. Brittle-to-Ductile Transition in Metallic Glass Nanowires. Nano Lett. 16, 4467-4471 (2016).

30. Shi, Y. Size-dependent mechanical responses of metallic glasses. Int. Mater. Rev. 0, 1-18 (2018).

31. Stukowski, A. Visualization and analysis of atomistic simulation data with OVITO-the Open Visualization Tool. Model. Simul. Mater. Sci. Eng. 18, 015012 (2010).

32. Falk, M. L. \& Langer, J. S. Dynamics of viscoplastic deformation in amorphous solids. Phys. Rev. E 57, 7192-7205 (1998). 


\section{FIGURES}

Initial morphology

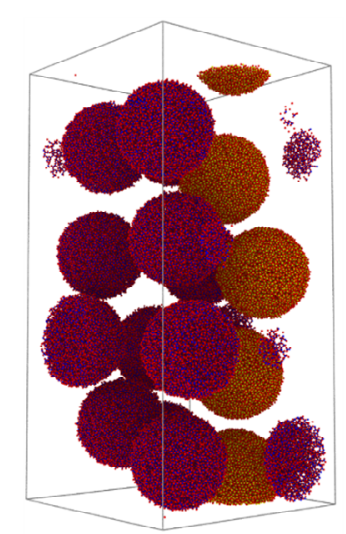

Final morphology

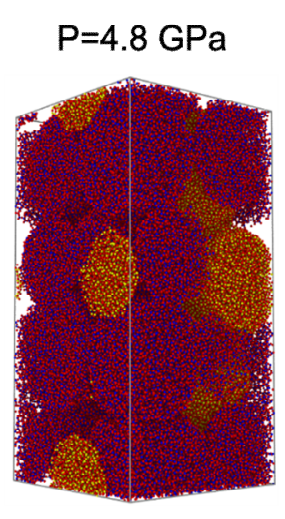

$$
\mathrm{P}=9.6 \mathrm{GPa}
$$

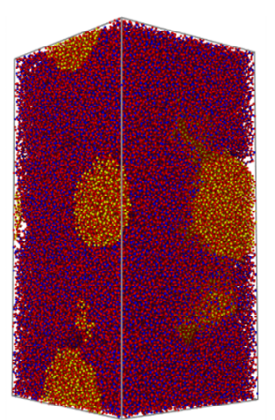

$\mathrm{P}=19.2 \mathrm{GPa}$

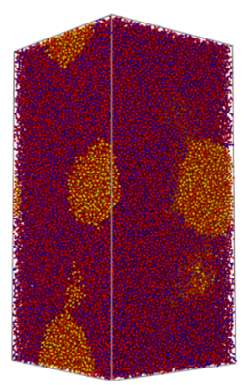

Figure 1 Illustration of starting nanoparticles and final morphologies consolidated at three representative pressure, the resulting nominal composition is $23.4 \mathrm{Al}_{2} \mathrm{O}_{3} 76.6 \mathrm{SiO}_{2}$. The size of nanoparticles is $8 \mathrm{~nm}$. The orange, purple nanoparticles represent amorphous alumina and silica, respectively. 


\section{BCS 3.2 GPa $300 \mathrm{~K} \quad$ BCS 19.2 GPa $300 \mathrm{~K} \quad$ BCS $3.2 \mathrm{GPa} 900 \mathrm{~K} \quad$ MCS $3.2 \mathrm{GPa} 300 \mathrm{~K}$}

(a)

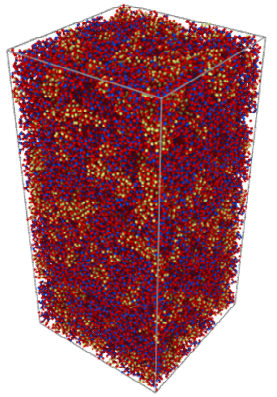

(b)

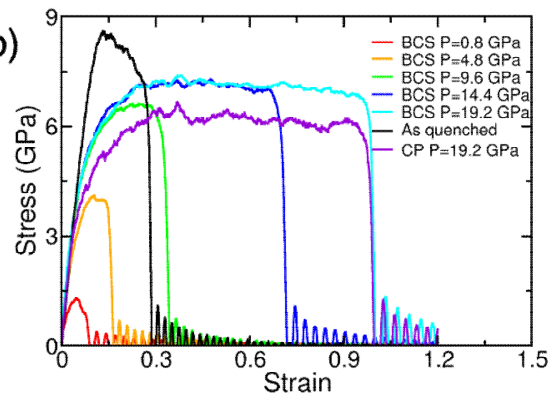

(d)

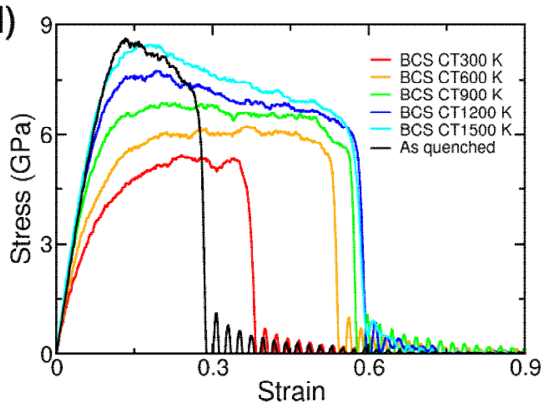

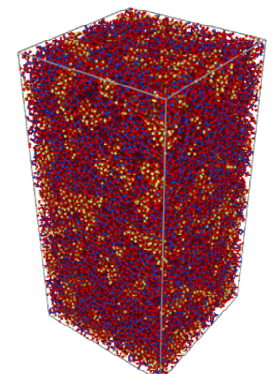

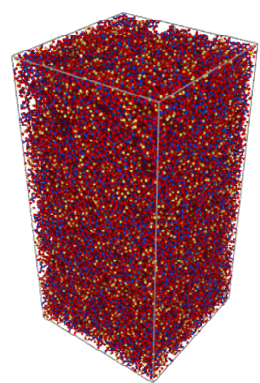

(c)

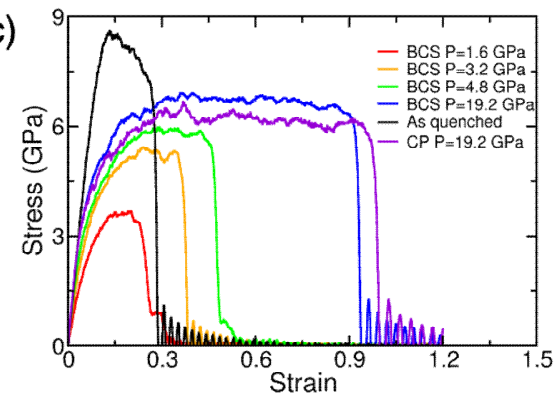

(e)

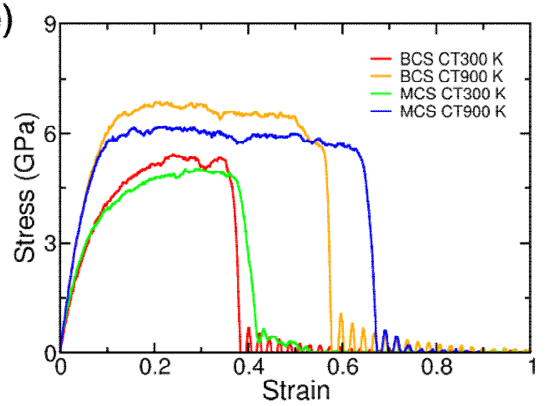

Figure 2 (a) The final morphologies of representative $23.4 \mathrm{Al}_{2} \mathrm{O}_{3} 76.6 \mathrm{SiO}_{2}$ glasses consolidated with different starting material at different conditions. BCS is prepared with a mixture of alumina and silica nanoparticles. MCS is prepared with $23.4 \mathrm{Al}_{2} \mathrm{O}_{3} 76.6 \mathrm{SiO}_{2}$ glassy nanoparticles. (b) stress-strain curves for $23.4 \mathrm{Al}_{2} \mathrm{O}_{3} 76.6 \mathrm{SiO}_{2}$ glasses consolidated at different pressure with 8-nm-glassy silica and alumina nanoparticles. (b) stress-strain curves for $23.4 \mathrm{Al}_{2} \mathrm{O}_{3} 76.6 \mathrm{SiO}_{2}$ glasses consolidated at different pressure with 1-nm-glassy silica and alumina nanoparticles. (c) stress-strain curves for $23.4 \mathrm{Al}_{2} \mathrm{O}_{3} 76.6 \mathrm{SiO}_{2}$ glasses consolidated at different temperature with 1-nm-glassy silica and alumina nanoparticles. The consolidation pressure is fixed at $3.2 \mathrm{GPa}$. (e) strain-stress curves of consolidated glass with different starting nanoparticle compositions. As-quenched glass and compressed bulk glasses $(\mathrm{CP})$ are shown here for comparison. 

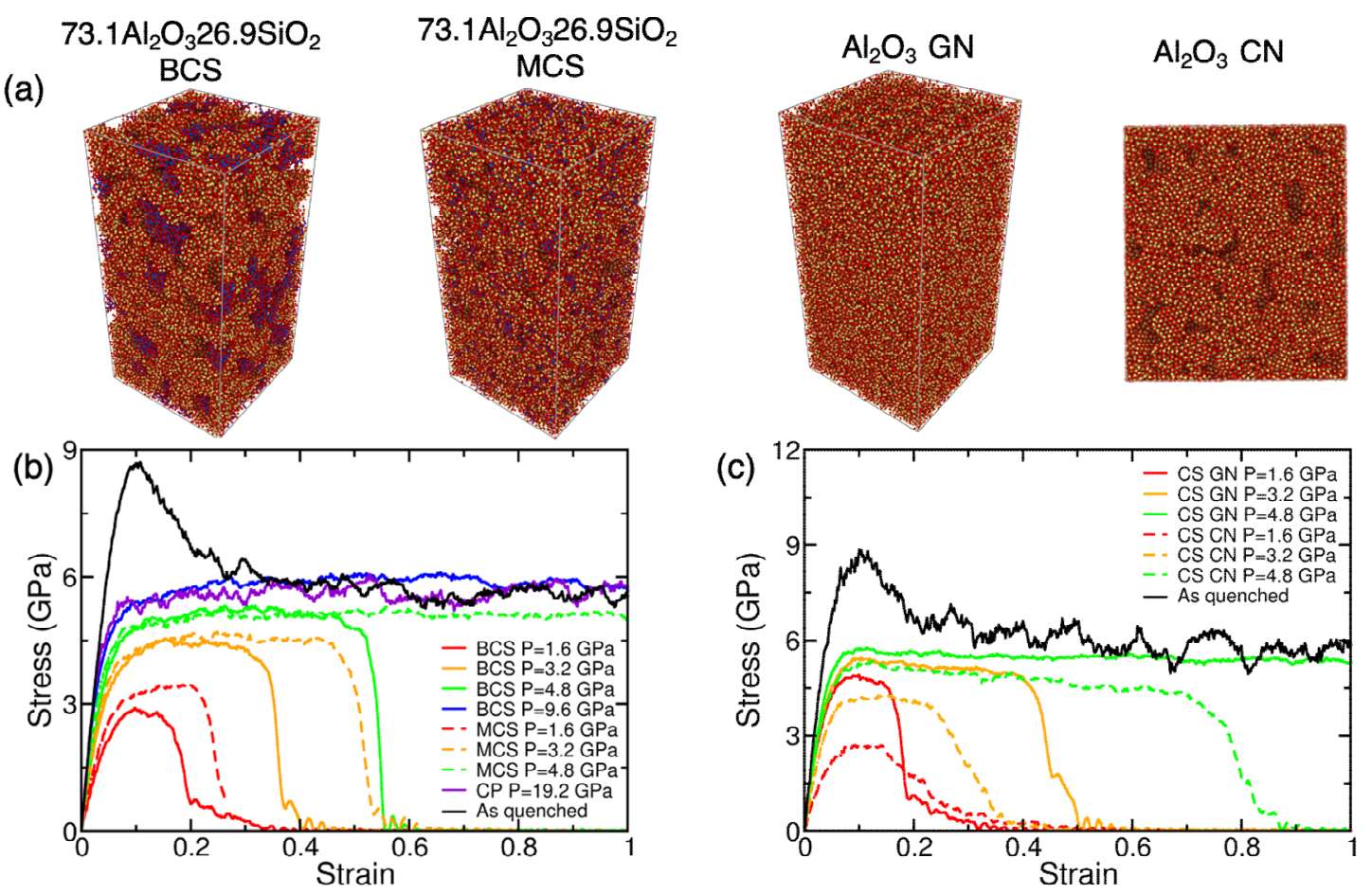

Figure 3 (a) The final morphologies of representative consolidated glasses prepared at room temperature under 3.2 GPa pressure. The BCS is prepared with a mixture of glassy alumina and silica nanoparticles. The MCS is prepared with $73.1 \mathrm{Al}_{2} \mathrm{O}_{3} 26.9 \mathrm{SiO}_{2}$ nanoparticles. Alumina consolidated with crystalline nanoparticles( $\mathrm{CN}$ ) show nanoscale crystal domains. (b) stress-strain curves for $73.1 \mathrm{Al}_{2} \mathrm{O}_{3} 23.9 \mathrm{SiO}_{2}$ glasses consolidated at different pressure. The solid lines are binary consolidated glasses with 2-nm glassy silica and alumina. The dash lines are mono consolidated glasses with 2-nm glassy $73.1 \mathrm{Al}_{2} \mathrm{O}_{3} 23.9 \mathrm{SiO}_{2}$ (c) stress-strain curves for pure alumina glasses consolidated at different pressure. The solid lines are consolidated with 2-nm glassy alumina. The dash lines are consolidated with 2-nm crystalline nanoparticles. As-quenched glass and compressed bulk glasses (CP) are shown here for comparison. 

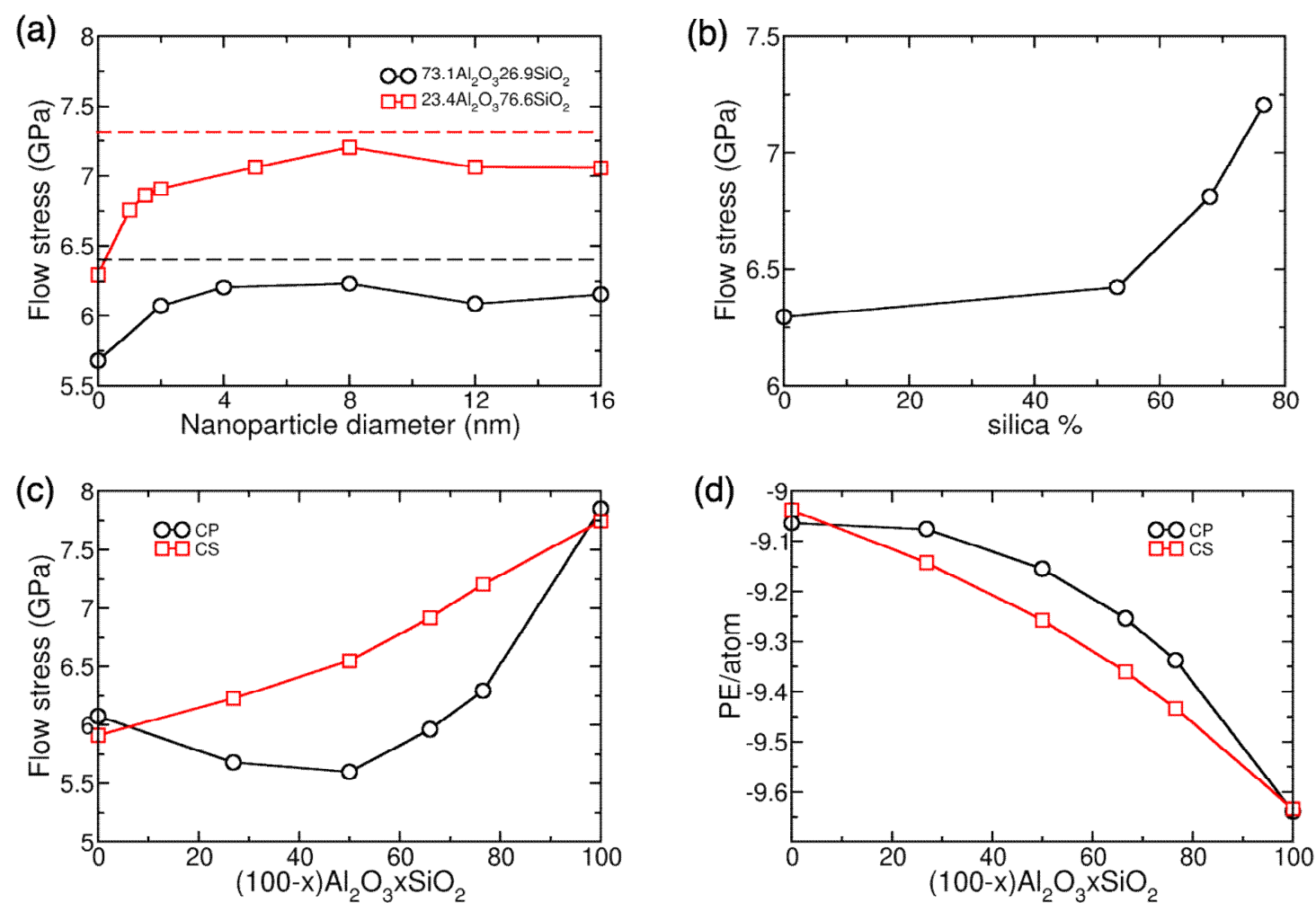

Figure 4 (a) The flow stress of consolidated glasses with as a function of starting nanoparticle size. The dash lines are the theoretical flow stress for two glassy systems. The consolidated glasses are prepared with a mixture of glassy silica and alumina. (b) The flow stress of $23.4 \mathrm{Al}_{2} \mathrm{O}_{3} 76.6 \mathrm{SiO}_{2}$ glass as a function of silica nanoparticle volume fraction. The nanoparticle size is fixed at $8 \mathrm{~nm}$. (c) Flow stress of glasses as a function of nominal compositions. CS is consolidated with 8-nm glassy silica and alumina. (d) The potential energy (PE) of atoms for consolidated glasses and compressed glasses as a function of nominal composition. Both CS and CP are consolidated at room temperature under 19.2 GPa pressure. The flow stress is averaged from $30 \%-65 \%$ strain. 

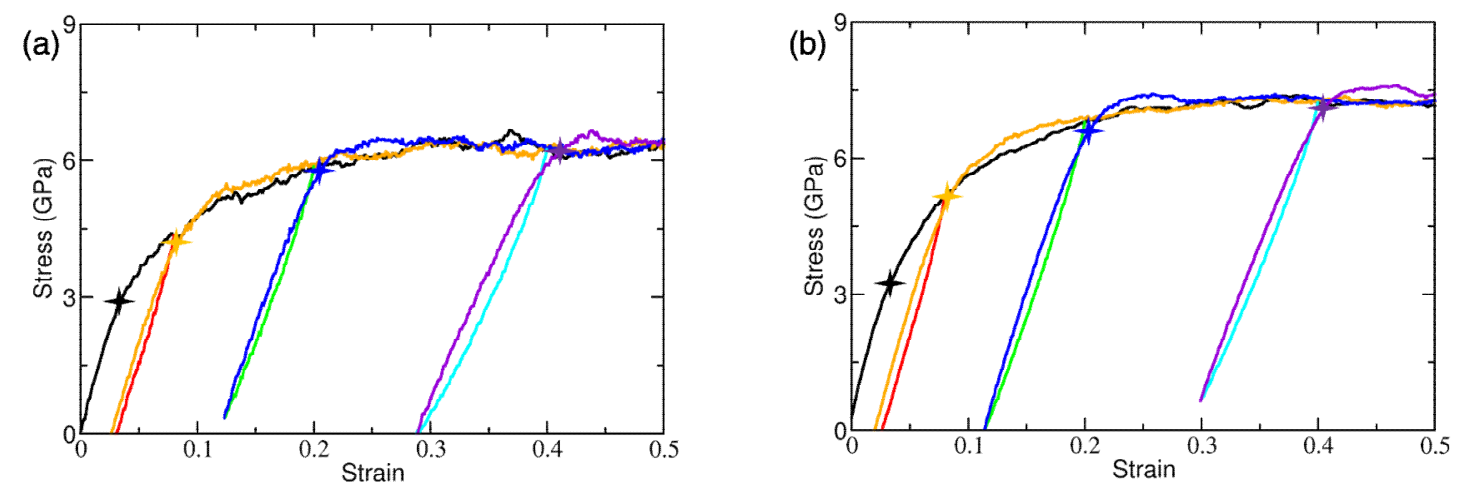

Figure 5 Work hardening of (a) compressed glass (b) consolidated glass with a nominal composition of $23.4 \mathrm{Al}_{2} \mathrm{O}_{3} 76.6 \mathrm{SiO}_{2}$. Consolidated glasses are prepared with 8-nm glassy silica and alumina. Both $\mathrm{CS}$ and $\mathrm{CP}$ are consolidated at room temperature with $19.2 \mathrm{GPa}$ pressure. The stars indicate the yield strength after cold working. 

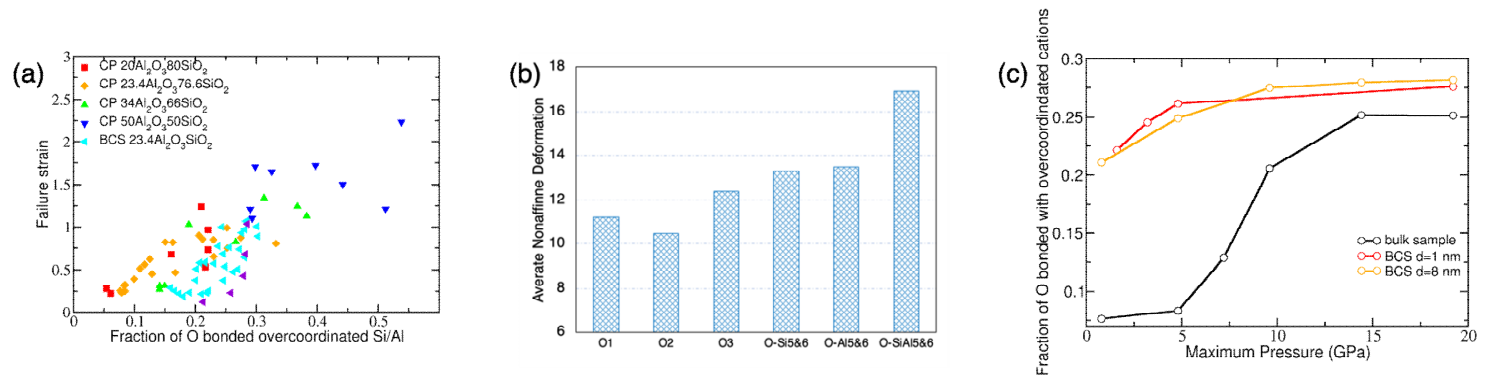

Figure 6 (a) Correlation between ductility and population of oxygen atoms bonded with overcoordinated cations for compressed glasses and consolidated glasses. Consolidated glasses are prepared with a mixture of glassy silica and alumina. Both CS and CP are prepared at difference temperature and pressure. (b) the rearrangement propensity of oxygen atoms with different bonding environments. $\mathrm{O} 1, \mathrm{O} 2, \mathrm{O} 3$ represent 1 fold,2-fold,3-fold oxygen atoms, while O-Si5\&6, O-A15\&6, O-SiA15\&6 represent oxygen atoms bonded with overcoordinated $\mathrm{Si}, \mathrm{Al}, \mathrm{Si}$ and $\mathrm{Al}$ atoms, respectively. (c) Population of oxygen atoms bonded with overcoordinated cations as a function of pressure for $23.4 \mathrm{Al}_{2} \mathrm{O}_{3} 76.6 \mathrm{SiO}_{2}$ prepared with different starting materials. 
के

(a)

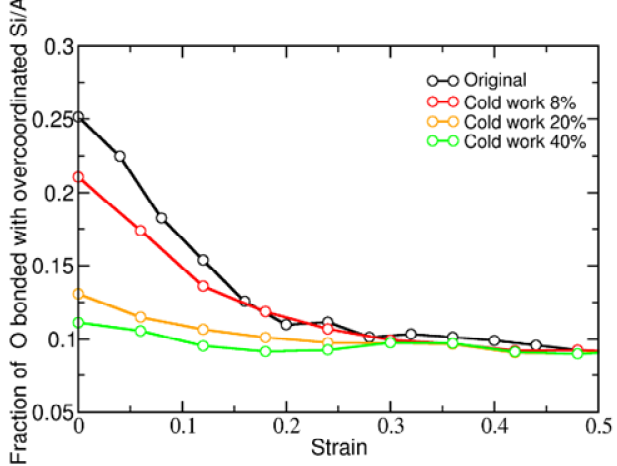

(b)

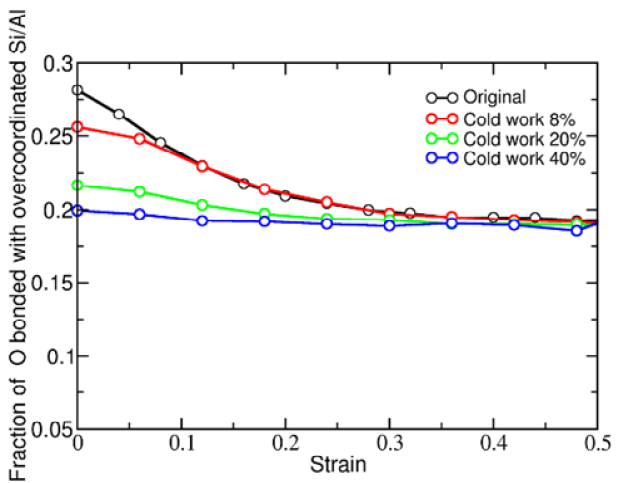

Figure 7 (a) population of oxygen atoms bonded with overcoordinated cations as a function of strain for compressed $23.4 \mathrm{Al}_{2} \mathrm{O}_{3} 76.6 \mathrm{SiO}_{2}$ glass after cold working. The compressed glass is prepared with $19.2 \mathrm{GPa}$ at room temperature before cold working. (b) population of oxygen atoms bonded with overcoordinated cations as a function of strain for consolidated $23.4 \mathrm{Al}_{2} \mathrm{O}_{3} 76.6 \mathrm{SiO}_{2}$ glass after cold working. The glass is consolidated with 8-nm amorphous silica and alumina under 19.2 GPa at room temperature. 


\section{Supporting Information}

Toward ultra-tough oxide glasses with heterogeneities by consolidation of nanoparticles

Yanming Zhang, Liping Huang, Yunfeng Shi* 
(a)

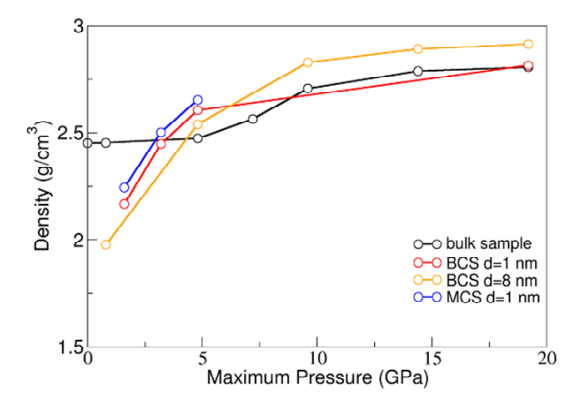

(b)

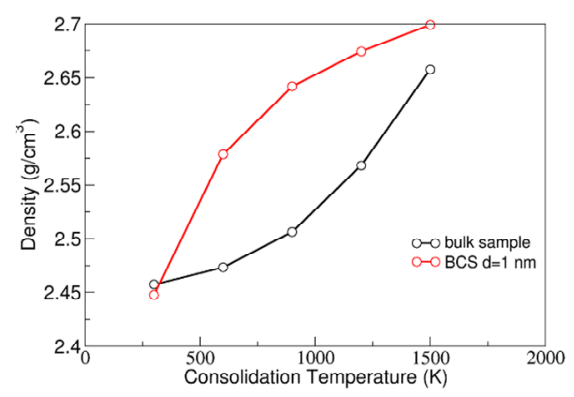

Figure $\mathrm{S} 1$ (a) ambient-condition density as a function of pressure for $23.4 \mathrm{Al}_{2} \mathrm{O}_{3} 76.6 \mathrm{SiO}_{2}$ glass prepared with different starting materials. The consolidation temperature is fixed at $300 \mathrm{~K}$. (b) ambient-condition density as a function of temperature for $23.4 \mathrm{Al}_{2} \mathrm{O}_{3} 76.6 \mathrm{SiO}_{2}$ glass prepared with different starting materials. The consolidation pressure is fixed at $300 \mathrm{~K}$. 


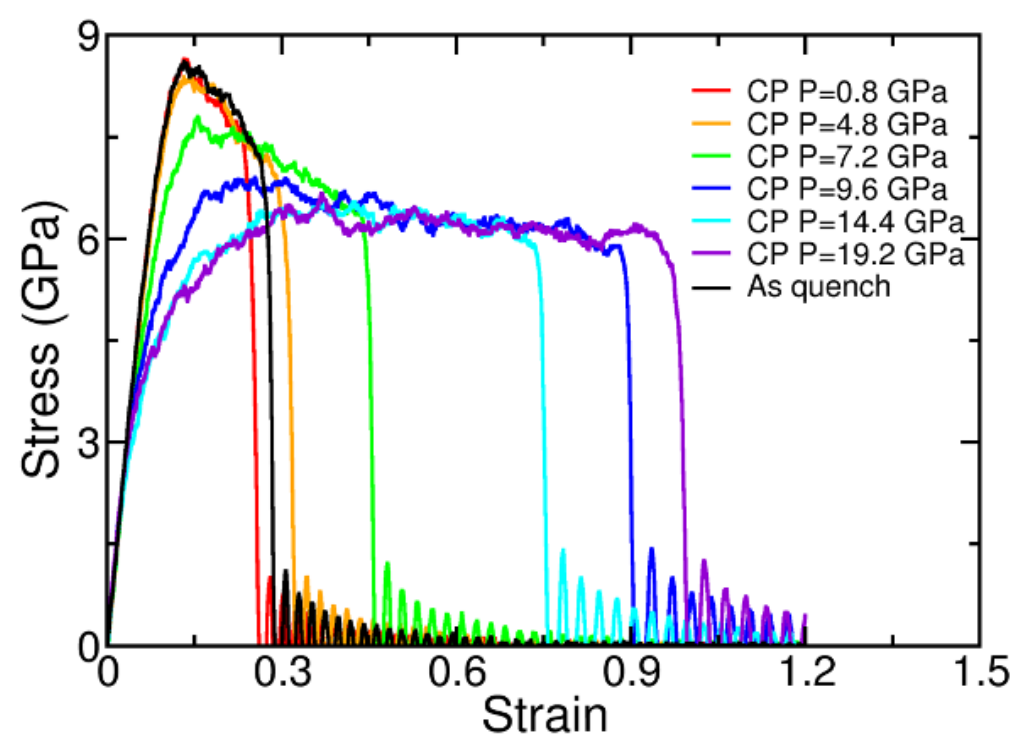

Figure S2 (a) strain-stress curves of compressed bulk $23.4 \mathrm{Al}_{2} \mathrm{O}_{3} 76.6 \mathrm{SiO}_{2}$ glasses prepared with different pressure at room temperature. (b) strain-stress curves of consolidated glass with different starting nanoparticle compositions. 

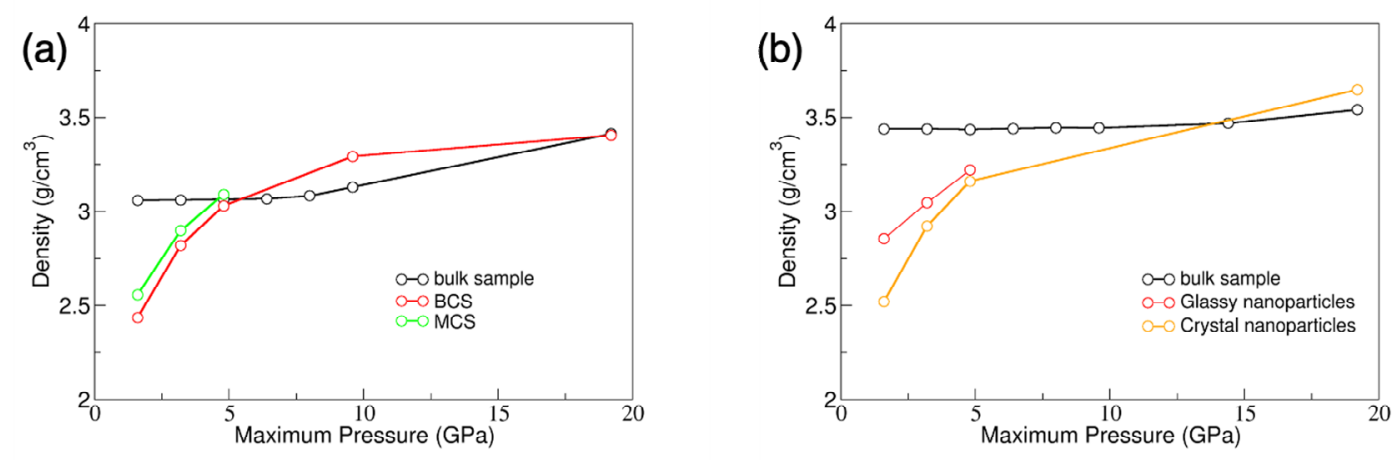

Figure $\mathrm{S} 3$ Ambient-condition density of (a) $73.1 \mathrm{Al}_{2} \mathrm{O}_{3} 26.9 \mathrm{SiO}_{2}$ (b) $\mathrm{Al}_{2} \mathrm{O}_{3}$ prepared with different starting materials under different pressure 

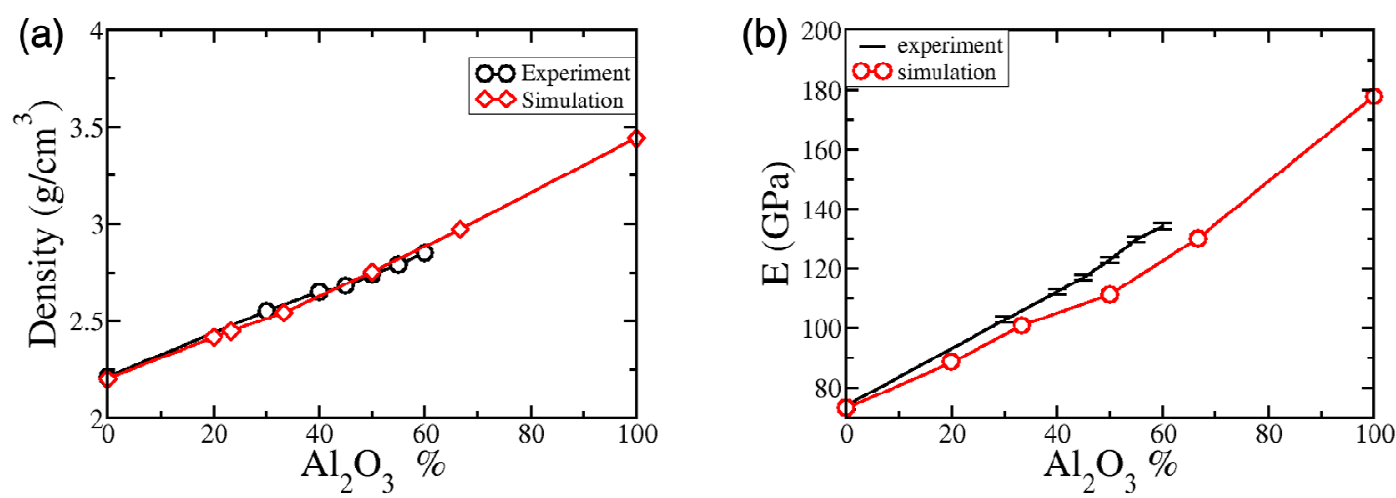

Figure S4 (a) Density (b) Young's moduli of binary aluminosilicate glasses in simulation as a function of compositions. Experimental measurements is included for comparison. 


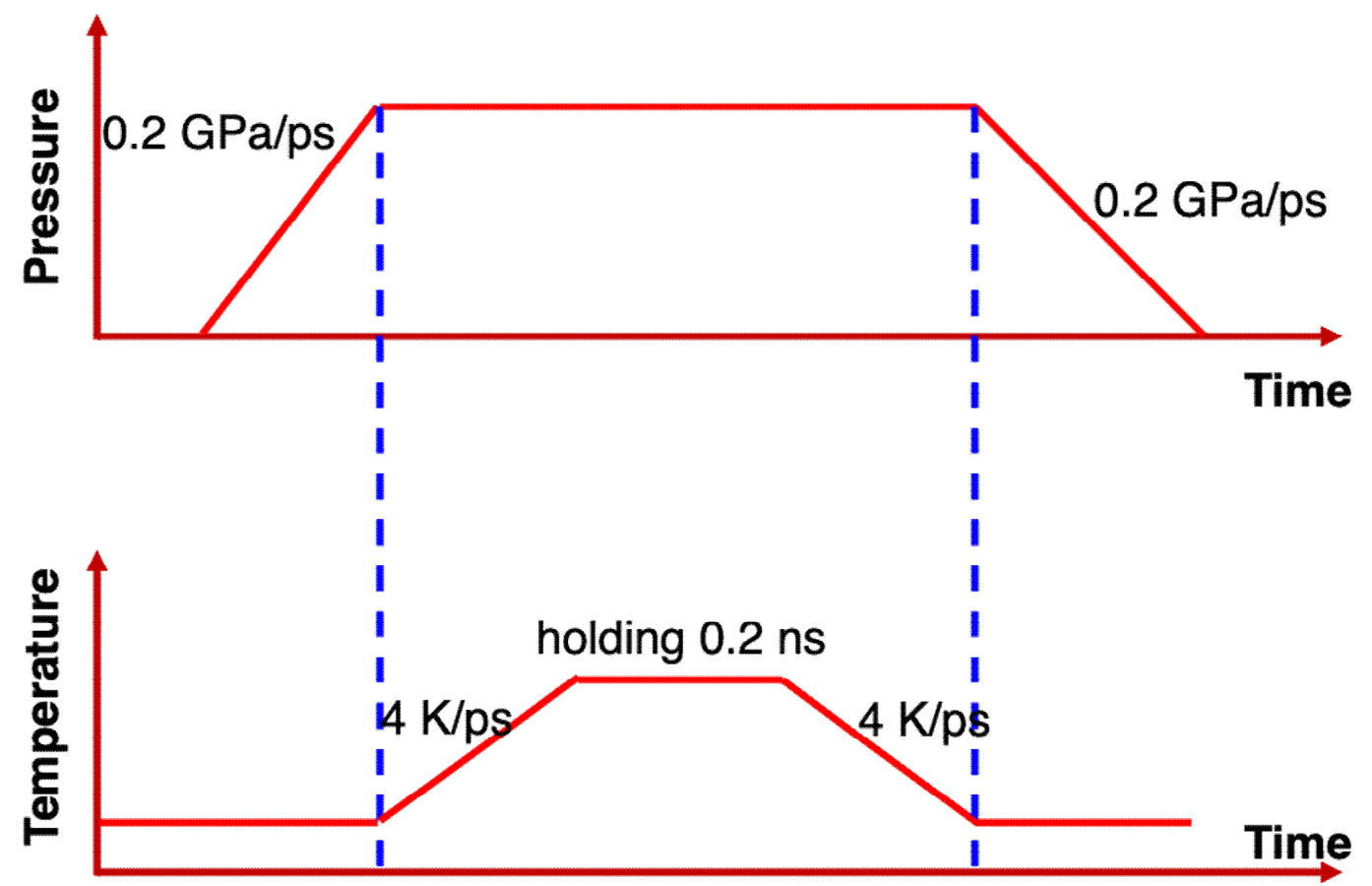

Figure S5 Temperature and pressure profile to consolidated oxide glasses. 
(a)

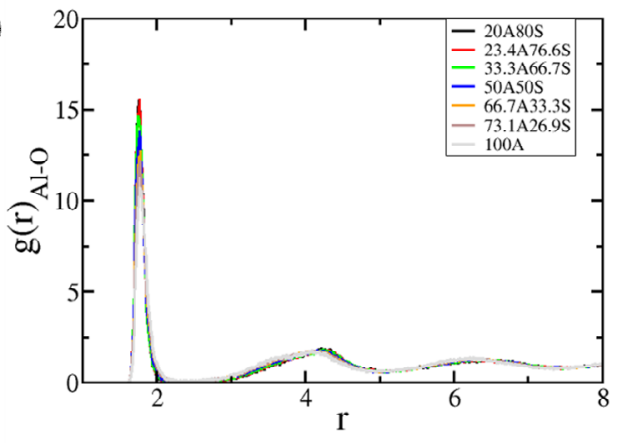

(c)

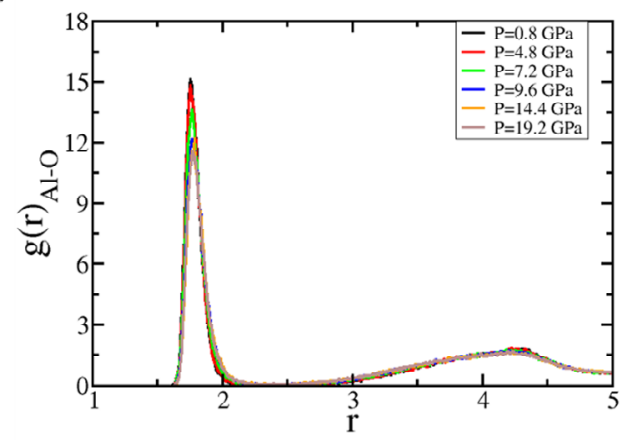

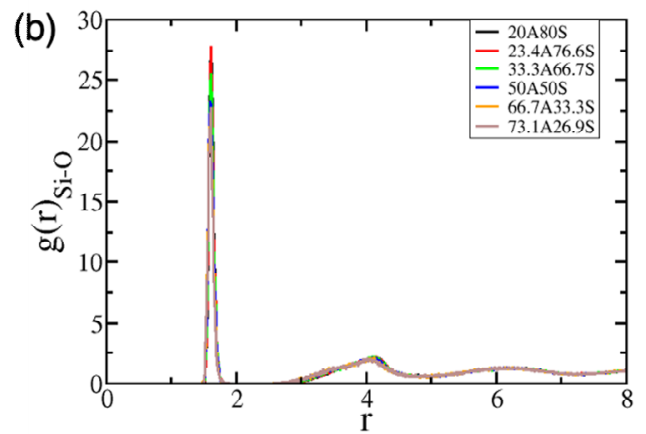

(d)

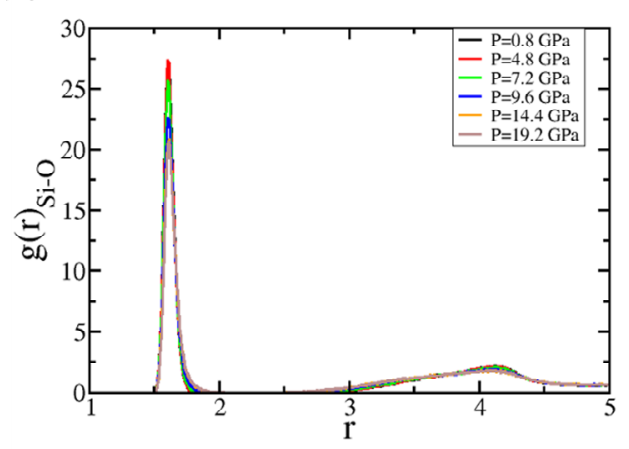

Figure S6 RDF of (a) Al-O (b) Si-O as a function of composition; RDF of (c) Al-O (d) Si-O as a function of pressure for compressed $23.4 \mathrm{Al}_{2} \mathrm{O}_{3} 76.6 \mathrm{SiO}_{2}$ glasses. The cutoff for Al-O, Si-O bond length is $0.24,0.22 \mathrm{~nm}$, respectively. 